\title{
The occurrence of infection-related systemic diseases in Korean children and adolescents has decreased after the spread of the COVID-19 pandemic: a multicenter retrospective study
}

\author{
Suk Jin Hong ${ }^{1} \wedge$, Ben Kang ${ }^{2} \wedge$, Jun Hyun Hwang ${ }^{3} \wedge$, Yu Bin Kim ${ }^{4} \wedge$, Yoo Min Lee ${ }^{5} \wedge$, Hyo-Jeong Jang ${ }^{6} \wedge$, \\ Kyung Jae Lee ${ }^{7} \wedge$, Soon Chul $\mathrm{Kim}^{8} \wedge$, Yunkoo Kang ${ }^{9} \wedge$, Hyun Jin $\mathrm{Kim}^{10} \wedge$, Ju Young $\mathrm{Kim}^{11} \wedge$, You Jin Choi ${ }^{12} \wedge$, \\ Eun Hye Lee ${ }^{13} \wedge$, So Yoon Choi ${ }^{14} \wedge$, Eunjoo Lee ${ }^{15} \wedge$, Byung-Ho Choe ${ }^{16} \wedge$, Soo Ahn Chae ${ }^{17} \wedge$, Sujin Choi ${ }^{2} \wedge$, \\ Won Suk Suh ${ }^{5 \wedge}$, Jeonglyn Song ${ }^{18 \wedge}$, Dae Yong $\mathbf{Y i}^{17 \wedge}$
}

${ }^{1}$ Department of Pediatrics, Daegu Catholic University School of Medicine, Daegu, Republic of Korea; ${ }^{2}$ Division of Pediatric Gastroenterology, Kyungpook National University Children's Hospital, School of Medicine, Kyungpook National University, Daegu, Republic of Korea; ${ }^{3}$ Department of Preventive Medicine, Daegu Catholic University School of Medicine, Daegu, Republic of Korea; ${ }^{4}$ Department of Pediatrics, Ajou University Medical Center, Suwon, Republic of Korea; ${ }^{5}$ Department of Pediatrics, Soonchunhyang University Bucheon Hospital, Soonchunhyang University College of Medicine, Bucheon, Republic of Korea; ${ }^{6}$ Department of Pediatrics, Keimyung University School of Medicine, Dongsan Medical Center, Daegu, Republic of Korea; ${ }^{7}$ Department of Pediatrics, Hallym University College of Medicine, Chuncheon, Republic of Korea; ${ }^{8}$ Department of Pediatrics, Jeonbuk National University Medical School and Hospital, Jeonju, Republic of Korea; ${ }^{9}$ Department of Pediatrics, Yonsei University Wonju College of Medicine, Wonju, Republic of Korea; ${ }^{10}$ Department of Pediatrics, Chungnam National University Hospital, Daejeon, Republic of Korea; ${ }^{11}$ Department of Pediatrics, Eulji University Hospital, Daejeon, Republic of Korea; ${ }^{12}$ Department of Pediatrics, Inje University Ilsan Paik Hospital, Goyang, Republic of Korea; ${ }^{13}$ Department of Pediatrics, Nowon Eulji Medical Center, Eulji University School of Medicine, Seoul, Republic of Korea; ${ }^{14}$ Department of Pediatrics, Kosin University Gospel Hospital, Kosin University College of Medicine, Busan, Republic of Korea; ${ }^{15}$ Department of Pediatrics, Haeundae Paik Hospital, Inje University College of Medicine, Busan, Republic of Korea; ${ }^{16}$ Department of Pediatrics, Kyunpook National University Hospital, School of Medicine, Kyungpook National University, Daegu, Republic of Korea; ${ }^{17}$ Department of Pediatrics, Chung-Ang University Hospital, College of Medicine, Chung-Ang University, Seoul, Republic of Korea; ${ }^{18}$ Chung-Ang University Industry Academic Cooperation Foundation, Seoul, Republic of Korea, Republic of Korea

Contributions: (I) Conception and design: SA Chae, DY Yi; (II) Administrative support: B Kang; (III) Provision of study materials or patients: All authors; (IV) Collection and assembly of data: DY Yi, J Song; (V) Data analysis and interpretation: JH Hwang, SJ Hong; (VI) Manuscript writing: All authors; (VII) Final approval of manuscript: All authors.

Correspondence to: Dae Yong Yi, MD, PhD. Division of Pediatric Gastroenterology and Hepatology, Department of Pediatrics, Chung-Ang University Hospital, 102, Heukseok-ro, Dongjak-gu, Seoul, 06973, Republic of Korea. Email: meltemp2@hanmail.net; Ben Kang, MD. Department of Pediatrics, School of Medicine, Kyungpook National University, 680 Gukchaebosang-ro, Jung-gu, Daegu 41944, Republic of Korea. Email: benkang@knu.ac.kr.

Background: The occurrences of infection-related systematic diseases, such as Henoch-Schönlein Purpura
(HSP), intussusception, and mucocutaneous lymph node syndrome (MCLS) may have decreased, similarly
to the decreased occurrence of infectious diseases following the Coronavirus disease 2019 (COVID-19)
pandemic. We aimed to investigate whether there was a change in the occurrence of these diseases in South
Korea after the spread of the COVID-19 pandemic.

Methods: In this multicenter, retrospective study conducted in 16 medical centers in South Korea

$\wedge$ ORCID: Suk Jin Hong, 0000-0003-4844-5044; Ben Kang, 0000-0002-8516-9803; Jun Hyun Hwang, 0000-0001-8547-0893; Yu Bin Kim, 0000-0001-6325-6191; Yoo Min Lee, 0000-0003-3554-6559; Hyo-Jeong Jang, 0000-0003-1496-5754; Kyung Jae Lee, 0000-0002-3969384X; Soon Chul Kim, 0000-0002-5947-4599; Yunkoo Kang, 0000-0003-1712-2138; Hyun Jin Kim, 0000-0003-0279-7925; Ju Young Kim, 0000-0002-4406-2428; You Jin Choi, 0000-0002-6882-3877; Eun Hye Lee, 0000-0002-9270-9783; So Yoon Choi, 0000-0002-7389-7678; Eunjoo Lee, 0000-0002-5351-5476; Byung-Ho Choe, 0000-0001-9899-9120; Soo Ahn Chae, 0000-0002-9825-8194; Sujin Choi, 00000001-8894-8127; Won Suk Suh, 0000-0001-5661-7876; Jeonglyn Song, 0000-0002-6578-6644; Dae Yong Yi, 0000-0002-4168-7131. 
patients diagnosed with HSP, intussusception, and MCLS at the age of <18 years between January 2016 and December 2020 were included. New occurrences of these three diseases were investigated monthly and annually, while to compare between the pre- and post-COVID-19 era cases, new occurrences between 2017-2019 and 2020 were compared. Additionally, the total annual occurrence rate was calculated by dividing each center's occurrence into the annual population per 100,000 of the population $<18$ years in each region that the center covers.

Results: A total 6,857 patients were included in this study. From 2017 to 2020, the number of patients diagnosed with HSP, intussusception, MCLS at the age of $<18$ years were 1,301, 1,693, and 3,863 patients, respectively. The average number of patients during the three years before the COVID-19 pandemic were each 379.7, 505.3, and 1,112.0 for HSP, intussusception, MCLS, respectively, which each decreased by $57.3 \%, 65.0 \%, 52.6 \%$ to $162,177,527$ in 2020, respectively. Furthermore, the total annual occurrence rate showed a significant decrease in 2020 compared to 2017 to 2019 in all three diseases (2017-2020; HSP: $11.85,12.96,10.52$, and 5.48; intussusception: 13.94, 16.97, 16.31, and 5.98; MCLS: 33.89, 35.11, 34.69, and 17.82 , respectively).

Conclusions: We revealed that the occurrence of HSP, intussusception, and MCLS, which are representative of infection-related systemic diseases in the pediatric population, decreased significantly after the spread of the COVID-19 pandemic.

Keywords: COVID-19; Henoch-Schönlein Purpura; intussusception; mucocutaneous lymph node syndrome

Submitted Jul 10, 2021. Accepted for publication Oct 07, 2021.

doi: $10.21037 /$ tp-21-315

View this article at: https://dx.doi.org/10.21037/tp-21-315

\section{Introduction}

It is likely that the year 2020 will be recorded on the pages of human history as a drastic year. Due to the Coronavirus disease 2019 (COVID-19) global pandemic, the daily lives of ordinary people were forced to change in many ways. This change was more direct in the healthcare sector, where they had no choice but to face the disease directly. Medical staff encountered many patients diagnosed with COVID-19, vaccines were developed for prevention, and efforts were made to cure infected patients. In addition to this direct impact of the COVID-19 infection, numerous medical staff and researchers worldwide conducted many studies related to the pandemic (1-3), such as research on its relationships with underlying diseases, treatment, prognosis, and most recently, vaccination.

However, other medical issues exist that are indirectly associated with the COVID-19 pandemic. Among these issues are the public awareness of the infection and increased enlightenment on the importance of personal hygiene, which is now demonstrated in everyday practice by social distancing, wearing masks, and washing hands (4). These new behaviors have decreased the development of various infectious diseases other than COVID-19 infections.
Furthermore, visits to medical institutions have decreased, which has also resulted in reduced medical costs (5-7). This tendency is also expected in South Korea, where the COVID-19 epidemic began early compared to other countries (8).

At this point, we were curious whether this reduced change in the occurrence of infectious diseases influenced by the COVID-19 pandemic was limited only to respiratory or gastrointestinal viral diseases. Based on literature, infection-related systematic diseases, such as Henoch-Schönlein Purpura (HSP), intussusception, and mucocutaneous lymph node syndrome (MCLS), are more likely to occur in children and adolescents than adults (9-11). HSP is generally known to occur in 10-30 patients per 100,000 under the age of 18 , and data from South Korea showed that HSP had occurred in approximately 17,000 patients from 2013 to $2016(9,12)$. Meanwhile, intussusception has been reported to occur in 9-328 per 100,000 , and approximately 25,000 patients have been reported in South Korea from 2008 to $2016(10,13)$. In the case of MCLS, there are regional differences in incidence, and the incidence in South Korea is approximately 217 per 100,000 population (14).

While limitations in explaining the direct causal 


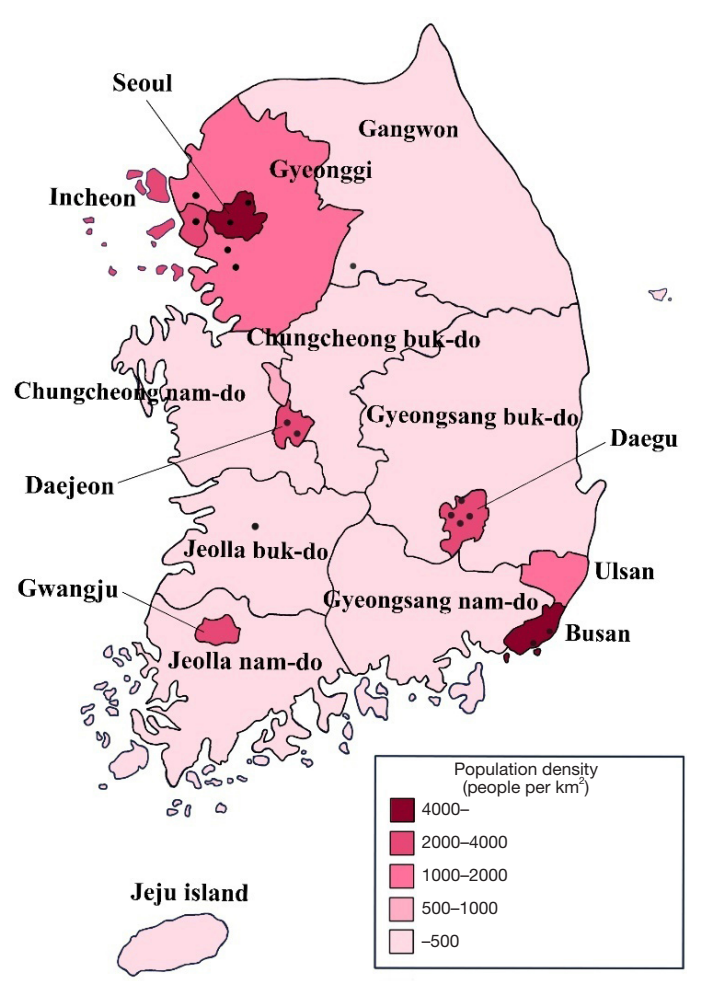

Figure 1 Geographic location of the participating centers on the map of South Korea.

relationship between these diseases and infections were observed, their associations with some pathogens have also been elucidated $(15,16)$. Currently, limited published data exist regarding the changes in the occurrence of these infection-related systematic diseases in the postCOVID-19 era. Therefore, in this study, we aimed to investigate whether there was a change in the occurrence of infection-related diseases, such as HSP, intussusception, and MCLS, in South Korea during the COVID-19 pandemic.

We present the following article in accordance with the STROBE reporting checklist (available at https://dx.doi. org/10.21037/tp-21-315).

\section{Methods}

\section{Patient selection}

This study was a multicenter, retrospective study conducted in 16 medical centers in South Korea (Figure 1). Patients included were those diagnosed with HSP (ICD 10 code: D690.02), intussusception (K561), and MCLS (M303) at the age of $<18$ years between January 2016 and December
2020. All participating centers were tertiary hospitals serving as referral centers in each region who also provided medical care for direct visits without referrals. Furthermore, medical charts were reviewed, and those whose diagnosis was indefinite were excluded. Additionally, patients who had previously been diagnosed with the same disease before the study period were also excluded. For this reason, data from 2016 were excluded, whereas, only data from January 2017 to December 2020 were included. New occurrences of these three diseases at the participating centers were investigated monthly and annually, while to compare between the preand post-COVID-19 era cases, new occurrences between 2017-2019 and 2020 were compared.

The rate of change was determined based on the first year to calculate changes in the number of patients each year for each disease. We also identified the average number of patients over the 3 years from 2017 to 2019, and the rate of change in 2020. The rate of changing patients' numbers was then calculated using the following equation:

The rate of change $(\%)=$ (patient number of target year

$$
\text { - patient number of the reference year) }
$$$$
\text { /patient number of reference year } \times 100
$$

Additionally, to identify the annual occurrence rate of these three diseases in each region, the population of people $<18$ years were applied using population census data obtained from Statistics Korea, which is a government organization for statistics under the Ministry of Economy and Finance (Table S1). Then, the total annual occurrence rate was calculated by dividing each center's occurrence into the annual population per 100,000 of the population $<18$ years in each region that the center covers. Thereafter, we summed the annual occurrence rate of each center.

\section{Statistical analysis}

To compare the fluctuations of the total annual occurrence rates over the years, a $95 \%$ confidence interval was calculated using the Clopper-Pearson method (17). Logistic regression analysis was performed to investigate the odds ratio (OR) for each year with 2020 as the reference. Data were considered statistically significantly different when the $\mathrm{P}$ value was $<0.05$. Statistical analysis was conducted using SAS software version 9.4 (SAS Institute, Cary, NC, USA).

\section{Ethics statement}

The study was conducted in accordance with the 


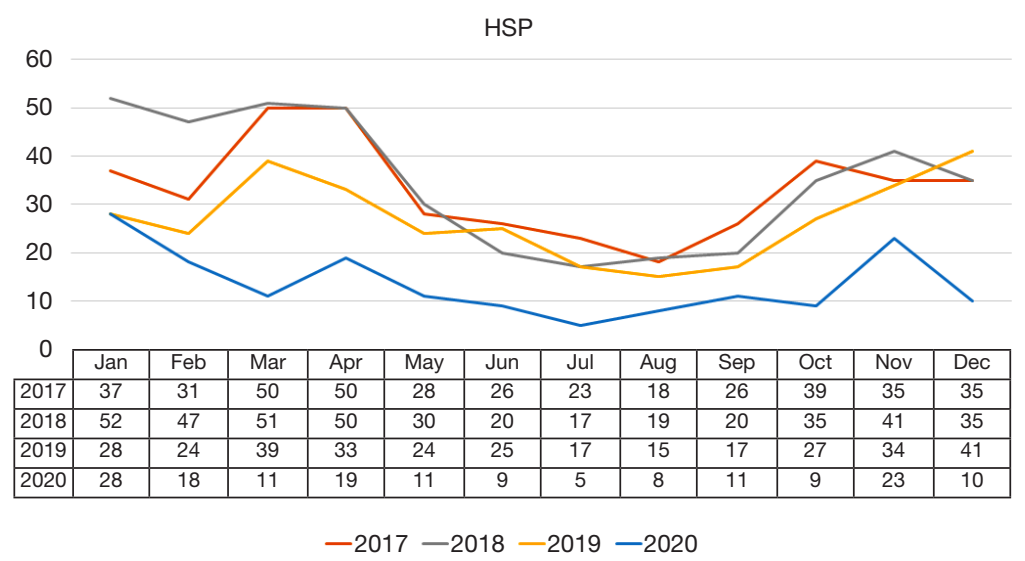

Figure 2 The occurrence of HSP before and after the spread of the COVID-19 pandemic. HSP, Henoch-Schönlein Purpura; COVID-19, Coronavirus disease 2019.

Declaration of Helsinki (as revised in 2013). The study was conducted with approval by the Institutional Review Board of 16 participating institutions including that of ChungAng University Hospital (IRB No.: 2012-020-19344) and individual consent for this retrospective analysis was waived.

\section{Results}

\section{Number of included patients}

A total of 6,857 patients were included in this study. From 2017 to 2020, the number of patients diagnosed with HSP, intussusception, MCLS at the age of $<18$ years in the participating centers were $1,301,1,693$, and 3,863 patients, respectively.

\section{Changes in the occurrence of HSP}

The number of patients diagnosed with HSP at the age of $<18$ years for the four years from 2017 to 2020 was 398, 417,324 , and 162, respectively (Figure 2). The average number of patients during the three years before the COVID-19 pandemic was 379.7, which decreased by $57.3 \%$ to 162 in 2020 . When comparing the monthly occurrence patterns between each year, the occurrence of HSP in each month decreased significantly in 2020 compared to the previous three years except in January, which was before the COVID-19 endemic started to spread in South Korea.

Furthermore, according to the Clopper-Pearson method, the total annual occurrence rates from 2017 to
2020 was 11.85 (95\% CI: 10.72-13.08), 12.96 (95\% CI: 11.74-14.26), 10.52 (95\% CI: 9.41-11.73), and 5.48 (95\% CI: 4.67-6.39) per 100,000 population, respectively (Figure 3). Logistic regression analysis revealed that the odds ratios of the total annual occurrence rate were each significantly higher in 2017-2019 compared to 2020, respectively (all $\mathrm{P}<0.001)$ (Table 1).

\section{Changes in the occurrence of intussusception}

The number of patients diagnosed with intussusception at the age of $<18$ years for the four years from 2017 to 2020 was $468,546,502$, and 177 , respectively (Figure 4). The average number of patients during the three years before the COVID-19 pandemic was 505.3, which decreased by $65.0 \%$ to 177 in 2020 . Furthermore, comparing the monthly occurrence of patterns between each year showed results similar to that of HSP. The occurrence of intussusception during each month also decreased significantly in 2020 compared to the previous three years except in January, which was before the COVID-19 endemic started to spread in South Korea.

Similarly, according to the Clopper-Pearson method, the total annual occurrence rates from 2017 to 2020 was 13.94 (95\% CI: 12.70-15.26), 16.97 (95\% CI: 15.57-18.45), 16.31 (95\% CI: 14.91-17.80), and 5.98 (95\% CI: 5.14-6.93) per 100,000 population, respectively (Figure 3). Logistic regression analysis revealed that the odds ratios of the total annual occurrence rate were each significantly higher in 2017-2019 compared to 2020, respectively (all $\mathrm{P}<0.001$ ) (Table 1). 


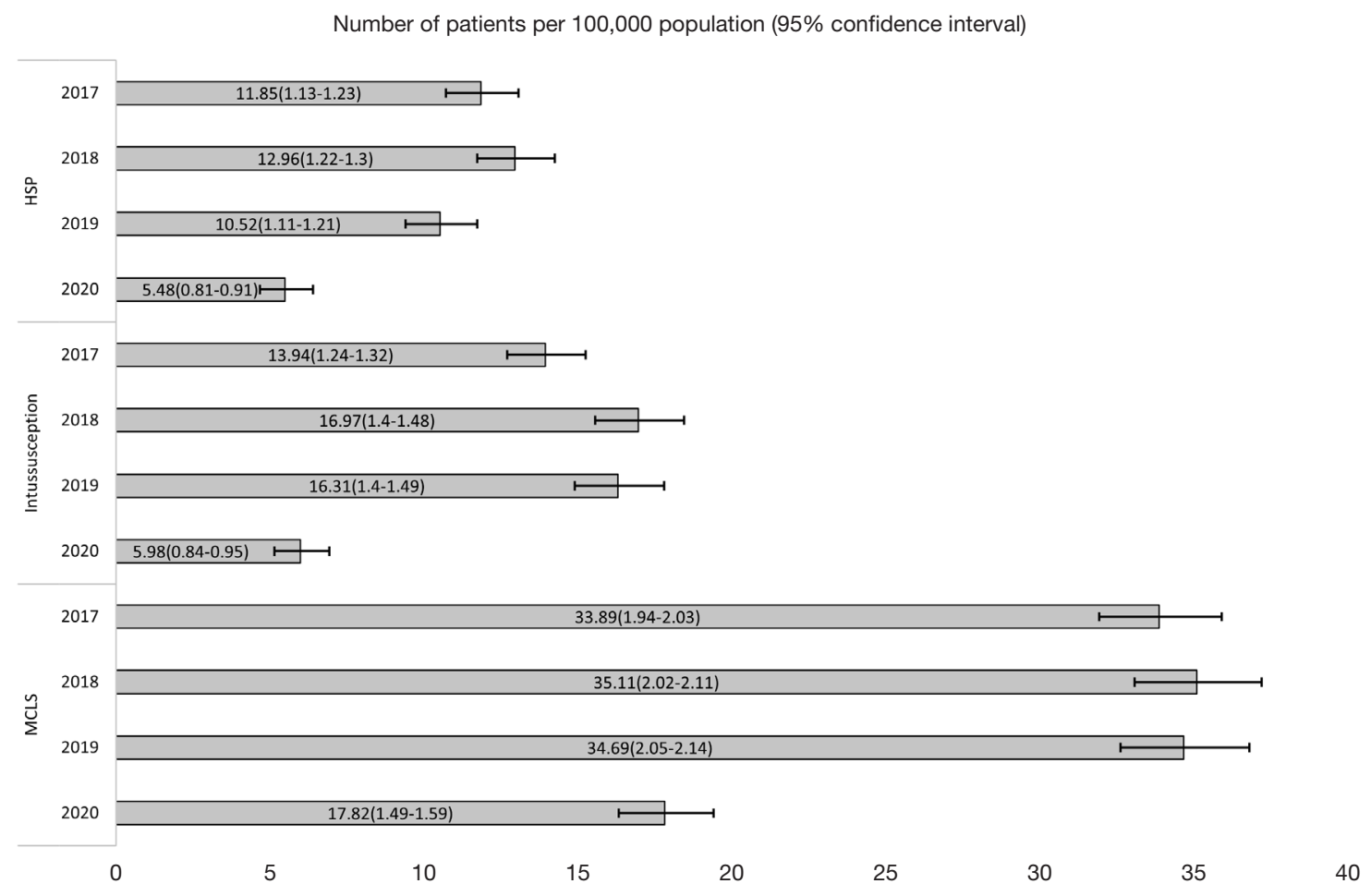

Figure 3 The total annual occurrence rate before and after the spread of the COVID-19 pandemic. COVID-19, Coronavirus disease 2019; HSP, Henoch-Schönlein Purpura; MCLS, mucocutaneous lymph node syndrome.

Table 1 Logistic regression analysis of each disease according to the occurrence rates by year

\begin{tabular}{lccc}
\hline Disease & Year & OR $(95 \% \mathrm{Cl})$ & $\mathrm{P}$ \\
\hline HSP & 2017 & $2.16(1.80-2.60)$ & $<0.001$ \\
& 2018 & $2.37(1.97-2.84)$ & $<0.001$ \\
& 2019 & $1.92(1.59-2.32)$ & $<0.001$ \\
& 2020 & Reference & \\
Intussusception & 2017 & $2.33(1.96-2.77)$ & $<0.001$ \\
& 2018 & $2.83(2.40-3.36)$ & $<0.001$ \\
& 2019 & $2.72(2.30-3.23)$ & $<0.001$ \\
& 2020 & Reference & \\
MCLS & 2017 & $1.90(1.72-2.11)$ & $<0.001$ \\
& 2018 & $1.97(1.78-2.19)$ & $<0.001$ \\
& 2019 & $1.95(1.75-2.16)$ & $<0.001$ \\
& 2020 & Reference &
\end{tabular}

HSP, Henoch-Schönlein Purpura; MCLS, mucocutaneous lymph node syndrome; OR, odds ratio; $\mathrm{Cl}$, confidence interval.

\section{Changes in the occurrence of mucocutaneous lymph node syndrome}

The number of patients diagnosed with MCLS at the age of $<18$ years for the four years from 2017 to 2020 was 1,138 , $1,130,1,068$, and 527, respectively (Figure 5). Furthermore, the average number of patients during the three years before the COVID-19 pandemic began was 1,112.0, which decreased by $52.6 \%$ to 527 in 2020 . Comparing the monthly occurrence patterns between each year showed results similar to that of HSP and intussusception. The occurrence of MCLS in each month decreased significantly in 2020 compared to the previous three years except in January and February, which was before the COVID-19 endemic started to spread in South Korea.

According to the Clopper-Pearson method, the total annual occurrence rates from 2017 to 2020 was 33.89 (95\% CI: 31.95-35.92), 35.11 (95\% CI: 33.09-37.22), 34.69 (95\% CI: 32.64-36.83), and 17.82 (95\% CI: 16.33-19.41) per 100,000 population, respectively (Figure 3). Logistic regression analysis revealed that the odds ratios of the total 


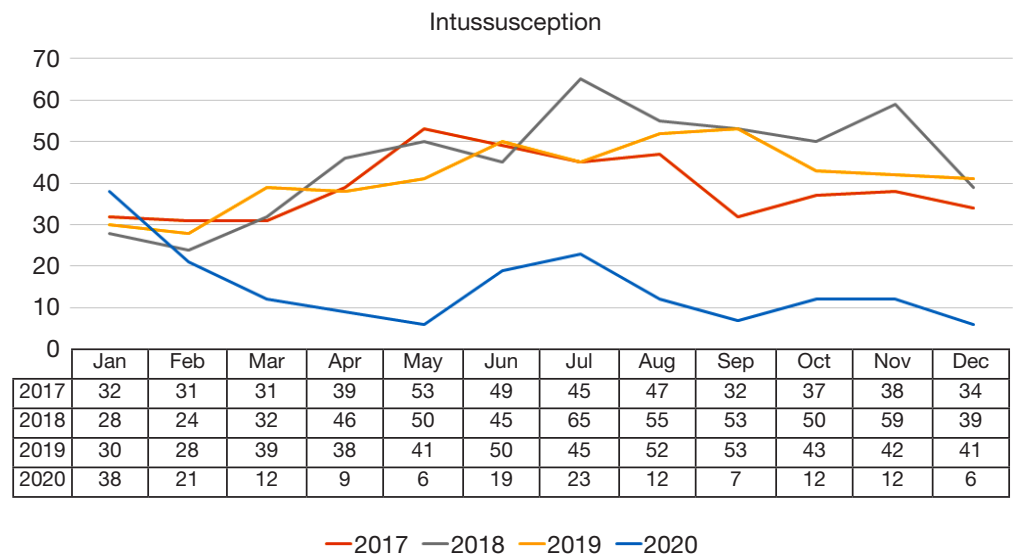

Figure 4 The occurrence of intussusception before and after the spread of the COVID-19 pandemic. COVID-19, Coronavirus disease 2019.

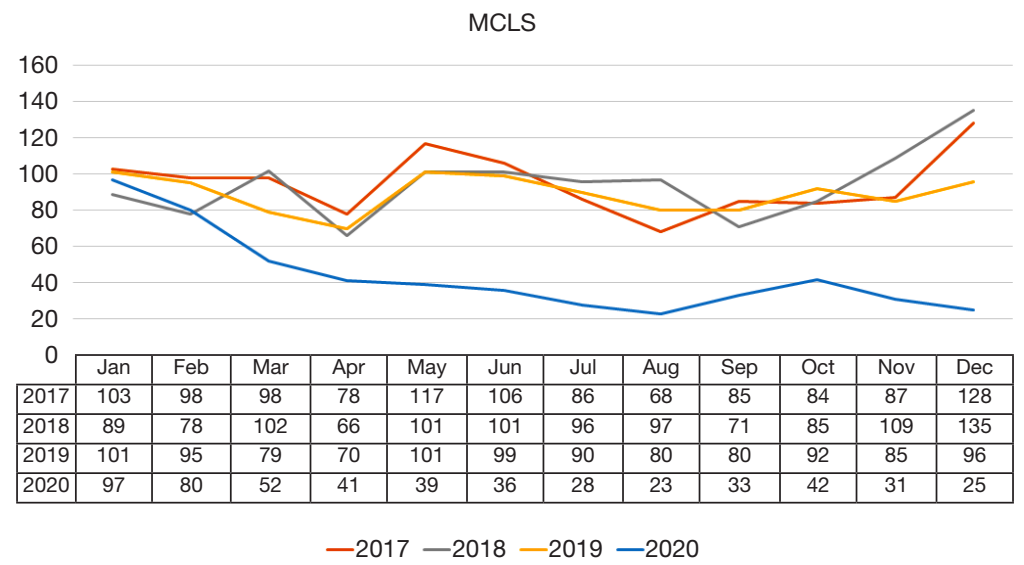

Figure 5 The occurrence of MCLS before and after the spread of the COVID-19 pandemic. MCLS, mucocutaneous lymph node syndrome; COVID-19, Coronavirus disease 2019.

annual occurrence rate were each significantly higher in 2017-2019 compared to 2020, respectively (all $\mathrm{P}<0.001$ ) (Table 1).

\section{Discussion}

This study is the first to identify the changes in occurrence patterns of infection-related systematic diseases in children and adolescents following the COVID-19 pandemic.

Through this nationwide study, we have identified that after the spread of the COVID-19 pandemic, the occurrence of infection-related systematic diseases, such as HSP, intussusception, and MCLS decreased by more than half compared to before. Furthermore, this pattern changes in infection-related systematic diseases, which reduced along with a decrease in infectious diseases, provide indirect evidence that the occurrences of these diseases were associated with a preceding infection. It has been elucidated in previous studies as well that these infection-related systematic diseases occurred during a particular season $(9,10,18-20)$. Therefore, although limitations still exist in explaining the direct causality or mechanisms, the associations between mycoplasma or influenza virus in HSP, adenovirus in intussusception, and a preceding respiratory illness in MCLS are well-known (14-16,21).

In South Korea, a rapid increase in the incidence of COVID-19 infections was observed in Daegu and North Gyeongsang Province between February and March 2020 when the pandemic had not yet spread to most Western countries. The second and third pandemic spread was also observed in summer and late 2020 in the densely populated 
Seoul and Gyeonggi Province. According to this study, we identified that regardless of the regional differences in COVID-19 incidence, the occurrence of these three diseases over the past year experienced a sharp decrease since February 2020. However, this decrease was indirectly related to the decrease in other infectious diseases following the COVID-19 pandemic, but is probably due to the increased awareness of COVID-19, thereby leading to the spread of strict personal hygiene management all over the country since early 2020. Similar patterns of this phenomenon were observed as well in the decreased occurrence of other diseases, including the decrease in hospital visits $(8,22)$.

Of course, there is a possibility that patients refrained from visiting hospitals despite symptoms that had occurred. A possibility exists also that treating other diseases at centers was difficult due to the COVID-19 pandemic. However, when considering the low medical fee and easy accessibility to medical institutions in South Korea, including the fact that most of the participating centers were not dedicated COVID-19 hospitals, it is unlikely that visits to these centers would have decreased for the aforementioned reasons. Moreover, considering the emergent medical condition of intussusception, ongoing associated long-term fever and various symptoms of MCLS, including purpura and complications in HSP, there is no doubt that the patient would have visited a medical institution (23).

Therefore, the reason why we considered these three diseases for the investigation was that these diseases show clear seasonality, and misdiagnosis seldomly occurs in tertiary medical settings. Our investigation of course cannot represent all patients in South Korea. However, all the centers that participated in the study were representative tertiary referral hospitals in each region, and limitations in treating these infection-related systematic diseases at primary medical institutions have been observed. Hence, it is likely that most of the patients with these diseases who resided within the region that the participating centers covered, would have visited that center. Therefore, the investigated data from each participating center are likely to have represented the incidence of infection-related systematic diseases in the region that center covers.

Furthermore, this study has some value as we compared only the changes in the number of cases; 3 years prior and 1 year after the start of the pandemic at the same centers, which are representative tertiary centers of the region.
Moreover, to minimize bias related to the change in the population, we applied the population number of residents under 18 years in the region and identified the occurrence rate. Finally, despite the dramatic decrease of infectious diseases over the past year, the relatively low decrease in the occurrence of these three diseases compared to infectious diseases implicated that the cause of these three diseases was not merely due to preceding infections.

Although, the occurrence of MCLS had decreased after the COVID-19 outbreak in South Korea according to the present study, there have been increasing reports of a Kawasaki disease-like, multisystem inflammatory syndrome in children infected with severe acute respiratory syndrome coronavirus 2 (SARS-CoV-2) $(3,24)$. This has been termed "Paediatric Multisystem Inflammatory Syndrome temporally associated with SARS-CoV-2 (PIMS-TS)", in the UK, or "Multisystem Inflammatory Syndrome in Children (MIS-C) associated with Coronavirus Disease 2019 (COVID-19)", in the US, although debate is still ongoing regarding the temporal association and nature of these hyper-inflammatory syndromes. Although no case of MIS-C associated with COVID-19 was reported in the present study, further investigations are required in this field as the COVID-19 pandemic continues. Moreover, because SARS-CoV-2 itself is a virus, there is a possibility of occurrence of infectionrelated systematic diseases after infection with SARS$\mathrm{CoV}-2$. A case of HSP has been reported after infection with SARS-CoV-2 (25).

This study has some limitations. First, as mentioned previously, this study did not include all the hospitals in South Korea. Therefore, it is hard to represent the general population of the country. Second, due to the retrospective nature of this study, some patients with the actual disease may have been excluded because of a misdiagnosis. Third, despite the fact that we not only analyzed the diagnostic codes but also investigated the electronical medical records of each patient, we may have missed some patients during this process. Therefore, caution is required when interpretating the results of this study. Future nationwide studies may better elucidate the changes in incidence of infection-related systemic diseases in the post-COVID-19 era.

In conclusion, we revealed that the occurrence of HSP, intussusception, and MCLS, which are representative of infection-related systemic diseases in the pediatric population, decreased significantly after the spread of the COVID-19 pandemic. These results also provide indirect evidence of their association with preceding infections. 


\section{Acknowledgments}

Funding: This work was supported by the National Research Foundation of Korea (NRF) grant funded by the Korean government (MSIT) (No. 2021R1A2C1011004) and granted to BK.

\section{Footnote}

Reporting Checklist: The authors have completed the STROBE reporting checklist. Available at https://dx.doi. org/10.21037/tp-21-315

Data Sharing Statement: Available at https://dx.doi. org/10.21037/tp-21-315

Peer Review File: Available at https://dx.doi.org/10.21037/tp21-315

Conflicts of Interest: All authors have completed the ICMJE uniform disclosure form (available at https://dx.doi. org/10.21037/tp-21-315). BK reports that this work was supported by the National Research Foundation of Korea (NRF) grant funded by the Korean government (MSIT) (No. 2021R1A2C1011004). The other authors have no conflicts of interest to declare.

Ethical Statement: The authors are accountable for all aspects of the work in ensuring that questions related to the accuracy or integrity of any part of the work are appropriately investigated and resolved. The study was conducted in accordance with the Declaration of Helsinki (as revised in 2013). The study was conducted with approval by the Institutional Review Board of 16 participating institutions including that of Chung-Ang University Hospital (IRB No.: 2012-020-19344) and individual consent for this retrospective analysis was waived.

Open Access Statement: This is an Open Access article distributed in accordance with the Creative Commons Attribution-NonCommercial-NoDerivs 4.0 International License (CC BY-NC-ND 4.0), which permits the noncommercial replication and distribution of the article with the strict proviso that no changes or edits are made and the original work is properly cited (including links to both the formal publication through the relevant DOI and the license). See: https://creativecommons.org/ licenses/by-nc-nd/4.0/.

\section{References}

1. Choi JY. COVID-19 in South Korea. Postgrad Med J 2020;96:399-402.

2. Choi SH, Kim HW, Kang JM, et al. Epidemiology and clinical features of coronavirus disease 2019 in children. Clin Exp Pediatr 2020;63:125-32.

3. Park SE. Epidemiology, virology, and clinical features of severe acute respiratory syndrome -coronavirus-2 (SARSCoV-2; Coronavirus Disease-19). Clin Exp Pediatr 2020;63:119-24.

4. Ha KM. The principle of distance during COVID-19 outbreak in Korea. Int Microbiol 2020;23:641-3.

5. Itaya T, Furuse $Y$, Jindai K. Does COVID-19 infection impact on the trend of seasonal influenza infection? 11 countries and regions, from 2014 to 2020. Int J Infect Dis 2020;97:78-80.

6. Karg MV, Alber B, Kuhn C, et al. SARS-CoV-2, influenza and norovirus infection: A direct epidemiologic comparison. Med Klin Intensivmed Notfmed $2021 \mathrm{Feb}$ 9:1-8. [Epub ahead of print].

7. Ahn SY, Park JY, Lim IS, et al. Changes in the Occurrence of Gastrointestinal Infections after COVID-19 in Korea. J Korean Med Sci 2021;36:e180.

8. Choi DH, Jung JY, Suh D, et al. Impact of the COVID-19 Outbreak on Trends in Emergency Department Utilization in Children: a Multicenter Retrospective Observational Study in Seoul Metropolitan Area, Korea. J Korean Med Sci 2021;36:e44.

9. Hwang HH, Lim IS, Choi BS, et al. Analysis of seasonal tendencies in pediatric Henoch-Schönlein purpura and comparison with outbreak of infectious diseases. Medicine (Baltimore) 2018;97:e12217.

10. Jo S, Lim IS, Chae SA, et al. Characteristics of intussusception among children in Korea: a nationwide epidemiological study. BMC Pediatr 2019;19:211.

11. McCrindle BW, Rowley AH, Newburger JW, et al. Diagnosis, Treatment, and Long-Term Management of Kawasaki Disease: A Scientific Statement for Health Professionals From the American Heart Association. Circulation 2017;135:e927-99.

12. Trnka P. Henoch-Schönlein purpura in children. J Paediatr Child Health 2013;49:995-1003.

13. Jiang J, Jiang B, Parashar U, et al. Childhood intussusception: a literature review. PLoS One 2013;8:e68482.

14. Kim GB. Reality of Kawasaki disease epidemiology. Korean J Pediatr 2019;62:292-6. 
15. Reamy BV, Williams PM, Lindsay TJ. Henoch-Schönlein purpura. Am Fam Physician 2009;80:697-704.

16. Jang J, Lee YJ, Kim JS, et al. Epidemiological Correlation between Fecal Adenovirus Subgroups and Pediatric Intussusception in Korea. J Korean Med Sci 2017;32:1647-56.

17. Abraham J. Computation of CIs for Binomial proportions in SAS and its practical difficulties. 2013. Available online: https://www.semanticscholar.org/paper/Computation-ofCIs-for-Binomial-proportions-in-SAS-Abraham/e0f243f4e5 ea664499bb1e0128c5ab8f4333114a. Accessed July 1, 2021.

18. Calviño MC, Llorca J, García-Porrúa C, et al. HenochSchönlein purpura in children from northwestern Spain: a 20-year epidemiologic and clinical study. Medicine (Baltimore) 2001;80:279-90.

19. Guo WL, Zhang SF, Li JE, et al. Association of meteorological factors with pediatric intussusception in subtropical china: a 5-year analysis. PLoS One 2014;9:e90521.

20. Uehara R, Belay ED. Epidemiology of Kawasaki disease in Asia, Europe, and the United States. J Epidemiol

Cite this article as: Hong SJ, Kang B, Hwang JH, Kim YB, Lee YM, Jang HJ, Lee KJ, Kim SC, Kang Y, Kim HJ, Kim JY, Choi YJ, Lee EH, Choi SY, Lee E, Choe BH, Chae SA, Choi S, Suh WS, Song J, Yi DY. The occurrence of infection-related systemic diseases in Korean children and adolescents has decreased after the spread of the COVID-19 pandemic: a multicenter retrospective study. Transl Pediatr 2021;10(11):2888-2896. doi: 10.21037/tp-21-315
2012;22:79-85.

21. Wang JJ, Xu Y, Liu FF, et al. Association of the infectious triggers with childhood Henoch-Schonlein purpura in Anhui province, China. J Infect Public Health 2020;13:110-7.

22. Wee LE, Venkatachalam I, Sim XYJ, et al. Containment of COVID-19 and reduction in healthcare-associated respiratory viral infections through a multi-tiered infection control strategy. Infect Dis Health 2021;26:123-31.

23. Lee EH, Yang HR. Nationwide Population-Based Epidemiologic Study on Childhood Intussusception in South Korea: Emphasis on Treatment and Outcomes. Pediatr Gastroenterol Hepatol Nutr 2020;23:329-45.

24. Yeo WS, Ng QX. Distinguishing between typical Kawasaki disease and multisystem inflammatory syndrome in children (MIS-C) associated with SARS-CoV-2. Med Hypotheses 2020;144:110263.

25. El Hasbani G, Taher AT, Jawad ASM, et al. HenochSchönlein purpura: Another COVID-19 complication. Pediatr Dermatol 2021. [Epub ahead of print]. 


\section{Supplementary}

Table S1 Annual population per 100,000 of the population <18 years in each region that the center covers

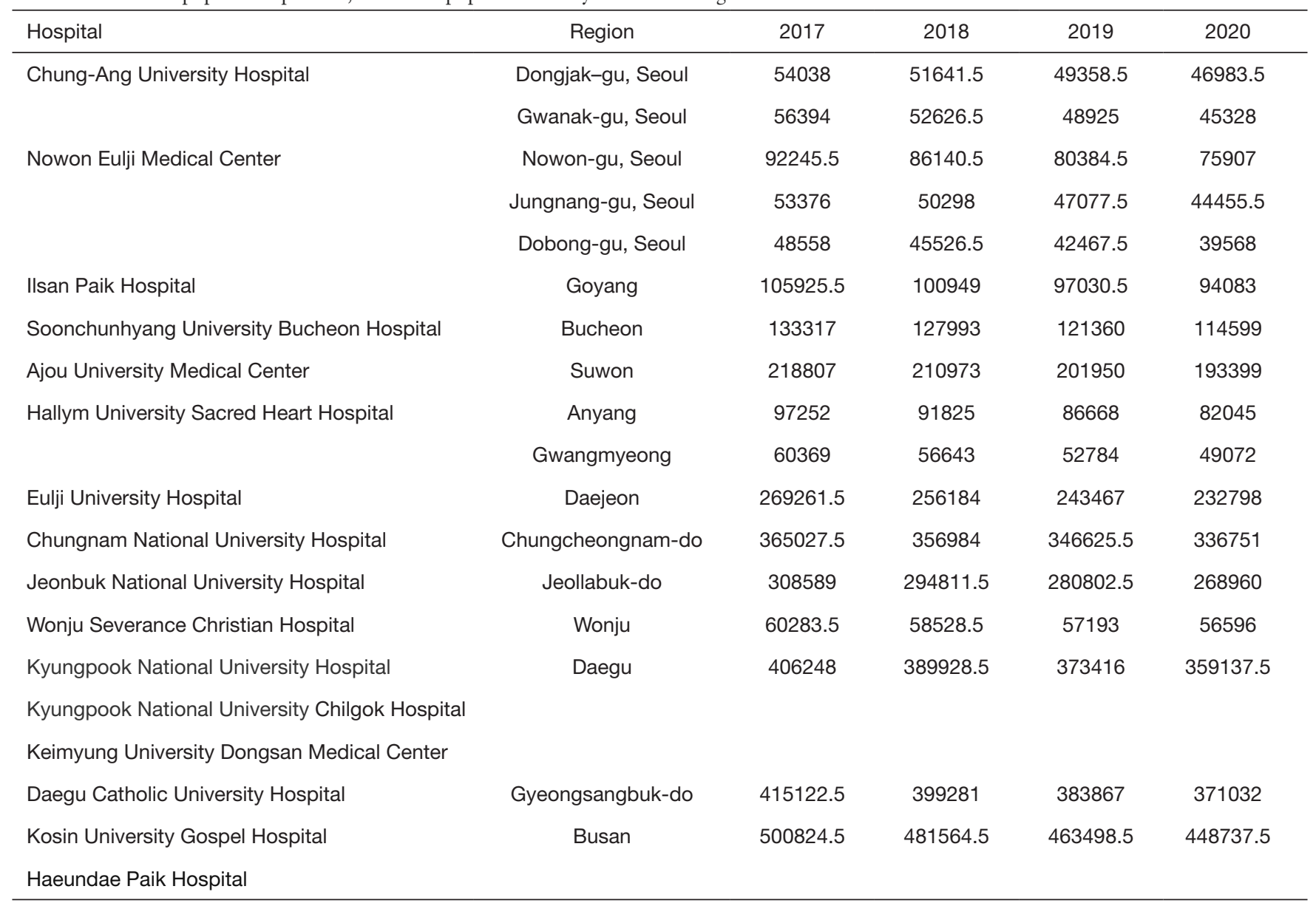

(c) Translational Pediatrics. All rights reserved. 